American Journal of Infectious Diseases 4 (2): 147-151, 2008

ISSN 1553-6203

(C) 2008 Science Publications

\title{
The Lipid-Lowering Efficacy of Switching Within Non-Nucleoside Reverse Transcriptase Inhibitors in HIV-Infected Patients
}

\author{
${ }^{1,2}$ A.M. Bain, ${ }^{1,2}$ K.D. Payne, ${ }^{1,2}$ A.P. Rahman, ${ }^{2,3}$ R. Bedimo, ${ }^{4,5}$ D.O. Maclayton, \\ ${ }^{5,6}$ M. Rodriguez-Barradas and ${ }^{1,2}$ A.J. Busti \\ ${ }^{1}$ School of Pharmacy Dallas/Forth Worth Regional Campus, \\ Texas Tech University Health Sciences Center, Dallas, Texas, USA \\ ${ }^{2}$ North Texas Veterans Affairs Healthcare System, Dallas, Texas, USA \\ ${ }^{3}$ The University of Texas Southwestern Medical Center, Dallas, Texas, USA \\ ${ }^{4}$ College of Pharmacy and Health Sciences, Texas Southern University, Houston, Texas, USA \\ ${ }^{5}$ Michael E. DeBakey Veterans Affairs Medical Center, Houston, Texas, USA \\ ${ }^{6}$ Baylor College of Medicine, Houston, Texas, USA
}

\begin{abstract}
The objective of present research is to evaluate the lipid lowering efficacy and safety of switching within non-nucleoside reverse transcriptase inhibitors (NNRTI) in HIV-infected patients. This is a multicenter, retrospective study utilizing a comprehensive electronic patient registry to identify all adult HIV-infected patients seen from October 1, 1998 through October 1, 2006, who substituted efavirenz for nevirapine $(\mathrm{EFV} \rightarrow \mathrm{NVP})$ or vice-versa $(\mathrm{NVP} \rightarrow \mathrm{EFV})$, without change in other antiretrovirals. Lipid profiles before and after the switch were analyzed. A total of 124 patients were identified with 14 male $(\mathrm{EFV} \rightarrow \mathrm{NVP}, \mathrm{n}=9$; $\mathrm{NVP} \rightarrow \mathrm{EFV}, \mathrm{n}=5)$ patients meeting the strict criteria for inclusion. An $\mathrm{EFV} \rightarrow \mathrm{NVP}$ switch resulted in significant reductions in TC $-16 \%$ and non-HDL $-25 \%$ $(\mathrm{p} \leq 0.02)$ and a trend towards a reduction in LDL-C $-12 \%, \mathrm{TG}-27 \%$, TC/HDL $-23 \%$, TG/HDL $-48 \%$ and an increase in HDL-C $+15 \%$ without any changes to BMI, viral or immunological control. However, a NVP $\rightarrow$ EFV switch appeared to result in a non-significant worsening of LDL-C $+29 \%$, HDL-C $-8 \%$, TG $+36 \%$, non-HDL $+28 \%$, TC/HDL $+57 \%$ and TG/HDL $+46 \%$. Lastly, more patients achieved their lipid goals when switched from EFV to NVP. These data suggest that switching from EFV to NVP-based HAART is associated with lipid improvement, however, switching from NVP to EFV-based HAART is associated with worsening of serum lipids.
\end{abstract}

Key words: Reverse transcriptase inhibitors, efavirenz, nevirapine, dyslipidemia, human immunodeficiency virus infection

\section{INTRODUCTION}

The widespread use of combination antiretroviral therapy (ART) has drastically improved the prognosis of patients with Human Immunodeficiency Virus (HIV). This improved prognosis has led to long-term use of antiretroviral agents, which have been associated with significant metabolic complications including dyslipidemia. Protease inhibitors (PI) have historically been considered the major cause of highly active antiretroviral therapy (HAART)-associated dyslipidemia $^{[1]}$. However, there is growing body of evidence associating clinically significant hyperlipidemia with non-nucleoside reverse transcriptase inhibitor (NNRTI)-based therapy, particularly with efavirenz (EFV)-based regimens ${ }^{[2-5]}$. Prospective comparisons of nevirapine (NVP) and EFV have demonstrated that EFV-based HAART is associated with greater elevation in total cholesterol (TC), low-density lipoprotein cholesterol (LDL-C), triglycerides (TG) and non-HDL cholesterol (nonHDL-C) as well as 1 increase in high-density lipoprotein (HDL-C) of lesser magnitude when compared to NVPbased HAART ${ }^{[4-6]}$. Antiretroviral switch strategies, in addition to traditional lipid-lowering treatment approaches, have proven to be useful in select patients with PI and thymidine analogue-associated dyslipidemia $^{[1,7-11]}$. Two studies have shown improvements in lipids when switching EFV to

Corresponding Author: Anthony J. Busti, Diplomate, Accreditation Council for Clinical Lipidology, Texas Tech University Health Sciences Center School of Pharmacy Dallas Fort Worth Regional Campus, 4500 S. Lancaster Rd, Bldg \#7, Dallas, Texas 75216, USA Tel: 214-372-5300/240 Fax: 214-372-5020 
$\mathrm{NVP}^{[12,13]}$. However, it is not known whether switching from NVP to EFV may worsen lipids. It is also unknown if intra-NNRTI switching translates into an increased number of patients achieving their patient specific lipid goals per the National Cholesterol Education Program (NCEP)/Adult Treatment Panel III (ATP III) guidelines in a real-world clinical practice setting $^{[13]}$. The present study was conducted to determine the effect of intra-NNRTI class switching on serum lipid and virologic parameters in HIV-infected patients on stable NNRTI-based HAART and to determine if this intervention translates into improvements in achievement of lipid goals.

\section{MATERIALS AND METHODS}

Study design, objectives and participants: This multicenter retrospective study evaluated serum lipid parameters in HIV-infected patients following a switch from one NNRTI-based regimen (either EFV or NVP) to another NNRTI-based therapy. The study was approved by all the Institutional Review Boards of the participating institutions.

Two electronic patient registries that contained comprehensive medical records were used to identify all HIV-infected patients seen at the Dallas and Houston Veterans Affairs Medical Centers during an eight-year period (October 1998 through October 2006). Patients were included if they were $\geq 18$ years of age, on a stable NNRTI-based HAART regimen for $\geq 4$ weeks prior to an isolated switch from EFV to NVP or from NVP to EFV and subsequently maintained on the second NNRTI-based regimen for $\geq 4$ weeks. They also had to have an on -treatment documented lipid profile obtained within a 6 month period prior to the switch and a follow-up lipid profile obtained within a 6 month period after switching to the new NNRTI based HAART. In an attempt to control for confounders strict exclusion criteria were used during screening. Patients were excluded if they had any concomitant changes in the nucleoside reverse transcriptase inhibitor (NRTI) backbone agents, any dosage change, additions or deletions of lipid-lowering medications or other medications known to alter serum lipid parameters, any documented evidence of significant changes in diet, alcohol-consuming behavior, exercise patterns or significant changes in diabetes control (defined as a change in $\mathrm{HbA} 1 \mathrm{c}>1 \%$ ). Patients with new-onset or uncontrolled thyroid disease or nephrotic syndrome were also excluded. All patients were risk stratified per NCEP/ATP III guidelines to determine whether or not patients achieved lipids goals pre and post switch.
Study endpoints: Changes in serum lipid profiles before and after the intra-NNRTI class switch were investigated as the primary efficacy measure while CD4 cell counts, HIV viral load and liver enzymes [aspartate aminotransferase (AST) and alanine aminotransferase (ALT)] data were collected at baseline and following the switch to evaluate the safety of the switch]. Secondary endpoints included investigation of changes in the total cholesterol to high-density lipoprotein ratio (TC/HDL) and the triglyceride to high-density lipoprotein ratio (TG/HDL) from baseline. We also assessed the frequency of NCEP/ATP III lipid goal attainment, both pre and post NNRTI switch.

Data analysis: At the time of protocol development, no data existed on the effects of any within NNRTI switch on the lipid profile and achievement of patient specific goals. Due to the limited nature of this intervention in this special population and available number of subjects, all patients in both HIV registries who meet the strict criteria for inclusion were included in this analysis. Median changes in serum lipids and CD4 counts from baseline were computed using the Wilcoxon Signed ranks test for paired continuous nonnormally distributed data. Frequency of NCEP/ATP III goal attainment and frequency of undetectable viremia were analyzed using McNemar's test. A two-sided alpha of 0.05 was used to determine statistical significance. All statistical analysis were performed using SPSS for Windows version 15.0 (SPSS Inc., Chicago, IL).

\section{RESULTS AND DISCUSSION}

A total of 124 patients were identified that had taken both NVP and EFV during an eight-year period with a total of 14 patients meeting the strict criteria for inclusion. Reasons for exclusion were based on a priori criteria and included incomplete lipid data, change of an NRTI backbone agent, change in lipid lowering medication and addition of a medication known to alter lipid parameters. The majority of patients had undetectable HIV viral loads (defined as $<50$ copies $\left.\mathrm{mL}^{-1}\right)$ at baseline $(\mathrm{n}=12 / 14)$ and only $1 / 14$ patients had a CD4 count $<200$ copies $\mathrm{mL}^{-1}$. Reasons patients were switched from EFV to NVP included adverse CNS side effects $(n=4)$, dyslipidemia $(\mathrm{n}=2)$, positive tetrahydrocannabinol (THC) screen $(\mathrm{n}=1)$ and reason unknown $(\mathrm{n}=2)$. Patients were switched from NVP to EFV for pill burden reduction $(n=2)$ or for other unknown reasons $(n=3)$. Baseline lipid data and other baseline characteristics are shown in Table 1 . 
Am. J. Infectious Dis., 4 (2): 147-151, 2008

Table 1: Baseline characteristics

\begin{tabular}{|c|c|c|}
\hline Characteristic & EFV to NVP $(n=9)$ & NVP to EFV $(n=5)$ \\
\hline Age (years) & $52(41-56)$ & $49(45-58)$ \\
\hline Males [n (\%)] & $9(100)$ & $5(100)$ \\
\hline HIV RNA Viral Load $<50$ copies $\mathrm{mL}^{-1}[\mathrm{n}(\%)]$ & $8(89)$ & $4(80)$ \\
\hline Median CD4+ counts $\left(\right.$ cells $\mathrm{mm}^{-3}$ ) & $506(319-621)$ & $517(295-622)$ \\
\hline BMI & $28(25-29)$ & $25(24-26)$ \\
\hline Duration of HIV (years) & $9(3-14)$ & $9(6-12)$ \\
\hline Duration on baseline NNRTI prior to switch (months) & $23(5-42)$ & $10(8-13)$ \\
\hline Thymidine analogue use in NRTI backbone [n (\%)] & $5(56) \mathrm{D} 4 \mathrm{~T}(\mathrm{n}=1)$ AZT $(\mathrm{n}=4)$ & $3(60)$ D4T $(n=1)$ AZT $(n=4)$ \\
\hline Time to follow-up lipid profile after NNRTI switch (weeks) & $9(4-16)$ & $14(11-20)$ \\
\hline \multicolumn{3}{|l|}{ Comorbidities: [n (\%)] } \\
\hline Diabetes & $1(11)$ & $1(11)$ \\
\hline Lipodystrophy & $1(11)$ & 0 \\
\hline Hyperlipidemia & $4(44)$ & $2(40)$ \\
\hline Coronary artery disease & $1(11)$ & 0 \\
\hline Hypothyroidism & $1(11)$ & 0 \\
\hline Use of lipid-lowering medications & $2(22)$ & 0 \\
\hline \multicolumn{3}{|l|}{ Lipid profile $\left(\mathrm{mg} \mathrm{dL}^{-1}\right)$} \\
\hline $\mathrm{TC}$ & $193(165-223)$ & $158(117-166)$ \\
\hline LDL-C & $116(96-124)$ & $64(50-78)$ \\
\hline HDL-C & $40(31-53)$ & $38(29-56)$ \\
\hline TG & $113(76-181)$ & $146(81-353)$ \\
\hline Non-HDL-C & $156(113-182)$ & $102(79-137)$ \\
\hline TG/HDL & $4.4(1.7-9.1)$ & $3.8(2.1-15.1)$ \\
\hline TC/HDL & $4.9(3.5-6.6)$ & $3.0(2.8-5.7)$ \\
\hline
\end{tabular}

All data reported as median \pm IQR unless otherwise stated. TC $=$ Total Cholesterol, LDL $=$ Low-density lipoprotein cholesterol, HDL $=$ Highdensity lipoprotein cholesterol, non-HDL $=$ Non-high-density lipoprotein cholesterol, $\mathrm{TG}=$ Triglycerides

Patients on EFV-based ART at baseline that were subsequently switched to and maintained on NVPbased ART experienced overall improvements in serum lipids with statistically significant reductions in TC and non-HDL from baseline values (Fig. 1A). Conversely, patients on NVP-based therapy who were switched to and maintained on EFV-based treatment experienced an overall worsening of lipid profiles, although this difference was not statistically significant (Fig. 1B).

In the $\mathrm{EFV} \rightarrow \mathrm{NVP}$ group, the number of patients meeting the NCEP/ATP III goal increased from $4(44 \%)$ to $6(67 \%)$ for LDL-C and from $5(55 \%)$ to $8(89 \%)$ for HDL-C and non-HDL cholesterol. However, there was no change from baseline in the number of patients meeting NCEP/ATP III lipid goals in the $\mathrm{NVP} \rightarrow \mathrm{EFV}$ group.

Regarding the safety of such an intervention, there were no significant changes in immunologic control. When switching from EFV to NVP there was no significant change in median CD4 count from 506 cells $\mathrm{mm}^{-3}$ (IQR, 319-621) at baseline versus 489 cells $\mathrm{mm}^{-3}$ (IQR, 329-681) at follow-up, $\mathrm{p}=0.93$. This was also true for those switching from NVP to EFV; CD4 count changed from 517 cells $\mathrm{mm}^{-3}$ (IQR, 295-622) at baseline to 492 cells $\mathrm{mm}^{-3}$ (IQR, 220-731) at follow-up, $p=0.50$. In addition, there were no changes in the percent of patients with undetectable HIV-RNA (87\% at baseline vs. 93\% at follow-up; $\mathrm{p}=1.00$ ) or hepatic transaminases $>2.5$ times the upper limit of normal $(0 \%)$ following a switch from EFV to NVP or from NVP to EFV.

To our knowledge, this is the first study to evaluate the alterations in lipid parameters following a switch from both EFV to NVP and from NVP to EFV. Present results suggest that overall improvements in lipids occur when switching from EFV to NVP, but not when switched from NVP to EFV. We found a statistically significant $16 \%$ reduction in TC and $25 \%$ reduction in non-HDL-C cholesterol and an overall improvement in TG, HDL-C, LDL-C and TC/HDL and TG/HDL, although the latter did not reach statistical significance. In either switch arm, there was no apparent compromise of virologic or immunologic control, at least in our population who had relatively good viral control at baseline.

These findings are consistent with previous reports that EFV-based regimens may be associated with more adverse metabolic effects and hyperlipidemia than NVP-based regimens ${ }^{[4-6]}$. This observation is also consistent with PI to NNRTI switch studies which demonstrate that switching from PI-based regimens to NVP-based therapy may offer greater lipid improvements than switching the PI component to $\mathrm{EFV}^{[1,7,9,14-17]}$. Taken together, these data highlight the important differences in the magnitude of dyslipidemia associated with EFV and NVP and would also suggest that the potential for an intra-NNRTI switch strategy for improvement of EFV-associated dyslipidemia may 
Am. J. Infectious Dis., 4 (2): 147-151, 2008



(A)

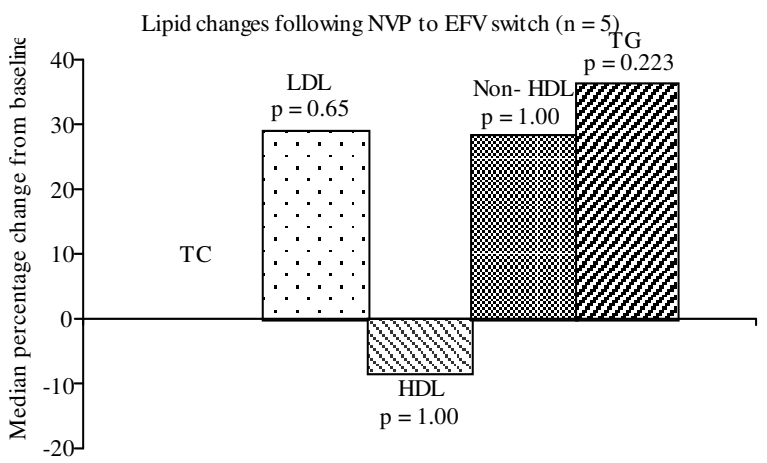

(B)

Fig. 1A and B: Changes in lipid parameters from baseline values following an intraNNRTI switch. TC $=$ Total cholesterol, $\mathrm{LDL}=$ Low-density lipoprotein cholesterol, HDL = High-density lipoprotein cholesterol, nonHDL = Non-high-density lipoprotein cholesterol, $\mathrm{TG}=$ Triglycerides

warrant further attention. Previous antiretroviral switch studies have also demonstrated maintenance of virologic and immunologic control if patients were well controlled on pre-switch antiretroviral regimens ${ }^{[6-11]}$. Our observations are consistent with one report that evaluated the lipid effects in patients who had experienced psychiatric side effects and were switched from $\mathrm{EFV}$ to $\mathrm{NVP}^{[12]}$. This retrospective study also found an overall improvement in TC, LDL-C, HDL-C and TG of $-9,-5,+7$ and $-33 \%$ respectively ${ }^{[12]}$. The reduction of LDL-C noted in this study is also consistent with the report by Parienti et al. ${ }^{[13]}$. They observed a modest reduction in LDL-C in patients who were switched from EFV-based therapy to NVP, when compared to patients who continued an EFV-based regimen $^{[13]}$.

Unlike the aforementioned studies that only explored a switch from EFV to NVP, we also evaluated the effect of switching from NVP to EFV. We observed a generalized worsening in lipid parameters and TC/HDL and TG/HDL in patients switched from NVP to $\mathrm{EFV}$, which help to support that any changes in lipids following the switch from EFV to NVP (or vice versa) are likely due to the switch and not potential confounding variables. A further investigation of the effect of an intra-NNRTI switch strategy on the attainment of NCEP goals was also conducted. While not statistically significant, a switch from EFV to NVP allowed an additional 2 patients (22\%) to attain their LDL-C goal and an additional 3 patients (33\%) to reach their HDL-C and non-HDL-C goals.

Some limitations to our study include its retrospective study design, small sample size and absence of females in our study population and inability to validate whether lipid profiles were obtained in the fasting state. However, this study used a comprehensive real-time database which allowed for controlling for numerous confounders that might affect the primary lipid outcomes, evaluating the effects on lipid parameters in both intra-NNRTI switches and the reporting the effect of such interventions on the attainment meaningful goals per current standards of care. In addition, it was based on real-world clinical practice from more than one health care center. Lastly, our study was conducted with a small sample size due to our strict criteria for inclusion and the limited number of patients who qualify for such a specific antiretroviral switch. However, this is also a limitation in much of the data in HIV-infected patients with dyslipidemia on HAART.

\section{CONCLUSIONS}

While targeting HAART-associated dyslipidemia has become increasingly important among HIVinfected patients as evidence accumulates demonstrating the high cardiovascular risk in this patient population, management of these lipid risk factors is often complicated and may require more than one intervention in order to attain lipid goals ${ }^{[1,2,18]}$. Consistent with other reports, these observations suggest that an intra-NNRTI switch from EFV to NVP is associated with global improvement of lipids, beyond decreases in LDL, without any readily apparent compromise in virologic or immunologic control. The ability of such a switch to impact lipid goal attainment may warrant further attention in order to determine if these lipid changes might also impact cardiovascular risk to an extent that is clinically meaningful. 


\section{REFERENCES}

1. Dube, M.P., J.H. Stein and J.A. Aberg et al., 2005. Guidelines for the evaluation and management of dyslipidemia in Human Immunodeficiency Virus (HIV)-infected adults receiving antiretroviral therapy: Recommendations of the HIV medicine association of the infectious disease society of america and the adult aids clinical trials group. Clin. Infect. Dis., 37: 613-627.

2. Friis Moller, N., R. Weber and P. Reiss et al., 2003. Cardiovascular disease risk factors in HIV patients- association with antiretroviral therapy. Results from the DAD study. AIDS., 17: 11791193.

3. Bonnet, F., M. Bonarek, S. DeWitte, J. Beylot and P. Morlat, 2002. Efavirenz-associated severe hyperlipidemia. Clin. Infect. Dis., 35: 776-777.

4. Manfredi, R., L. Calza and F. Chiodo, 2005. An extremely different dysmetabolic profile between the two available nonnucleoside reverse transcriptase inhibitors: Efavirenz and nevirapine. J. Acquir. Immune Defic. Syndr., 38 :236-238.

5. Van Leth, F., P. Phanuphak and K. Ruxrungtham, 2004. Comparison of first-line antiretroviral therapy with regimens including nevirapine, efavirenz, or both drugs, plus stavudine and lamivudine: A randomized, open-label trial, the 2NN study. Lancet., 363: 1253-1263.

6. Fontas, E., F. van Leth and C.A. Sabin et al., 2004. Lipid profiles in HIV-infected patients receiving combination antiretroviral therapy: Are different drugs associated with different lipid profiles? J. Infect. Dis., 189: 1056-1074.

7. Fisac, C., E. Fumero and M. Crespo et al., 2005. Metabolic benefits 24 months after replacing a protease inhibitor with abacavir, efavirenz, or nevirapine. AIDS., 19: 917-925.

8. Negredo, E., J. Ribalta and R. Paredes et al., 2002. Reversal of atherogenic lipoprotein profile in HIV1 infected patients with lipodystrophy after replacing protease inhibitors by nevirapine. AIDS., 16: 1383-1389.

9. Calza, L., R. Manfredi and V. Colangeli et al., 2005. Substitution of nevirapine or efavirenz for protease inhibitor versus lipid-lowering therapy for the management of dyslipidemia. AIDS., 19: 1051-1058.
10. Llibre, J.M., P. Domingo and R. Palacios et al., 2006. Sustained improvement of dyslipidemia in HAART-treated patients replacing stavudine with tenofovir. AIDS., 20: 1407-1414.

11. Gatell, J., D. Salmon-Ceron and A. Lazzarin et al., 2007. Efficacy and safety of atazanavir-based highly active antiretroviral therapy in patients with virologic suppression switched from a stable, boosted or unboosted protease inhibitor treatment regimen: The SWAN study (AI424-097) 48-week results. Clin. Infect. Dis., 44: 1484-1492.

12. Ward, D. and J.M. Curtin, 2006. Switch from efavirenz to nevirapine associated with resolution of efavirenz-related neuropsychiatric adverse effects and improvement in lipid profiles. AIDS Patient Care STDs., 20: 542-548.

13. Parienti, J., V. Massari, D. Rey, P. Poubeau and R. Verdon, 2007. Efavirenz to nevirapine switch in HIV-1-Infected patients with dyslipidemia: A randomized, controlled study. Clin. Infect. Dis., 45: 263-266.

14. Negredo, E., J. Ribalta and R. Ferre et al., 2004. Efavirenz induces a striking and generalized increase of HDL-cholesterol in HIV-infected patients. AIDS, 18: 819-821.

15. Negredo, E., J. Ribalta and R. Paredes et al., 2002. Reversal of atherogenic lipoprotein profile in HIV1 infected patients with lipodystrophy after replacing protease inhibitors by nevirapine. AIDS, 16: 1383-1389.

16. Martinez, E., I. Conget, L. Lozano, R. Casamitjana and J.M. Gatell, 1999. Reversion of metabolic abnormalities after switching from HIV-1 protease inhibitors to nevirapine. AIDS, 13: 805-810.

17. Ruiz, L., E. Negredo and P. Domingo et al., 2001. Antiretroviral treatment simplification with nevirapine in protease inhibitor experienced patients with HIV-associated lipodystrophy: 1-year prospective follow-up of a multicenter, randomized, controlled study. J. Acquir. Immune Defic. Syndr, 27: 229-236.

18. Triant, V.A., H. Lee, C. Hadigan and S.K. Grinspoon, 2007. Increased acute myocardial infarction rates and cardiovascular risk factors among patients with HIV disease. J. Clin. Endocrinol. Metab., 92: 2506-2512. 